\title{
Diastolic dysfunction is associated with an increased risk of contrast-induced nephropathy: a retrospective cohort study
}

Hyang Mo Koo, Fa Mee Doh, Kwang II Ko, Chan Ho Kim, Mi Jung Lee, Hyung Jung Oh, Seung Hyeok Han, Beom Seok Kim, Tae-Hyun Yoo, Shin-Wook Kang and Kyu Hun Choi*

\begin{abstract}
Background: Contrast-induced nephropathy $(\mathrm{CIN})$ is the third leading cause of hospital-acquired acute kidney injury, and it is associated with poor long-term clinical outcomes. Although systolic heart failure is a well-known risk factor for CIN, no studies have yet evaluated the association between diastolic dysfunction and CIN.

Methods: We conducted a retrospective study of 735 patients who underwent percutaneous transluminal coronary angioplasty (PTCA) and had an echocardiography performed within one month of the procedure at our institute, between January 2009 and December 2010. CIN was defined as an increase of $\geq 0.5 \mathrm{mg} / \mathrm{dL}$ or $\geq 25 \%$ in serum creatinine level during the 72 hours following PTCA.

Results: CIN occurred in 64 patients (8.7\%). Patients with CIN were older, had more comorbidities, and had an intra-aortic balloon pump (IABP) placed more frequently during PTCA than patients without CIN. They showed greater high-sensitivity C-reactive protein (hs-CRP) levels and lower estimated glomerular filtration rates (eGFR). Echocardiographic findings revealed lower ejection fraction and higher left atrial volume index and $E / E^{\prime}$ in the CIN group compared with non-CIN group. When patients were classified into 3 groups according to the E/E' values of 8 and 15, CIN occurred in $42(21.6 \%)$ patients in the highest tertile compared with $20(4.0 \%)$ in the middle and 2 (4.3\%) in the lowest tertile $(\mathrm{p}<0.001)$. In multivariate logistic regression analysis, E/E' $>15$ was identified as an independent risk factor for the development of CIN after adjustment for age, diabetes, dose of contrast media, IABP use, eGFR, hs-CRP, and echocardiographic parameters [odds ratio (OR) 2.579, 95\% confidence interval (Cl) 1.082-5.964, $\mathrm{p}=0.035]$. In addition, the area under the receiver operating characteristic curve of E/E' was 0.751 $(95 \% \mathrm{Cl} 0.684-0.819, \mathrm{p}<0.001)$, which was comparable to that of ejection fraction and left atrial volume index (0.739 and 0.656 , respectively, $\mathrm{p}<0.001)$.
\end{abstract}

Conclusions: This study demonstrated that, among echocardiographic variables, E/E' was an independent predictor of CIN. This in turn suggests that diastolic dysfunction may be a useful parameter in CIN risk stratification.

Keywords: Contrast-induced nephropathy, Diastolic dysfunction, E/E'

\footnotetext{
* Correspondence: khchoi6@yuhs.ac

Department of Internal Medicine, College of Medicine, Yonsei University, 134

Shinchon-dong, Seodaemun-gu, Seoul, Korea
} 


\section{Background}

Contrast-induced nephropathy (CIN) is one of the principal complications which develop after procedures using contrast media $(\mathrm{CM})$. Although there are some differences between institutions, the most commonly used definition of CIN in clinical trials is a rise in serum creatinine of $>0.5 \mathrm{mg} / \mathrm{dL}$ or $>25 \%$ from the baseline value within 72 hours after exposure to the $\mathrm{CM}$. The incidence of CIN is reported to be about $2 \%$ in the general population, $7-15 \%$ in patients undergoing percutaneous coronary intervention, whereas it increases up to $50 \%$ in high-risk patients with diabetes (DM) and renal failure $[1,2]$. Recently, risk stratification and application of preventive methods against CIN further reduced the incidence of CIN. However, CIN is still an important problem in that dependence for medical procedures using $\mathrm{CM}$ is steadily increasing and that it is closely related with poor long-term clinical outcomes: longer hospital stay, and increased mortality and post-procedural cardiovascular complications $[3,4]$.

Accordingly, many investigators have tried to reveal the pathogenesis of CIN. Delineated mechanisms include intra-renal vasoconstriction, reduced renal blood flow, medullary hypoxia, oxidative stress, inflammation, endothelial dysfunction, and direct tubular-epithelial cell injury by $\mathrm{CM}$ [5]. At the same time, risk factors potentiating the processes explained above were proved out: renal failure, old age, DM, congestive heart failure (CHF), hypotensive event, use of intra-aortic balloon pump (IABP), and ionic/ high-osmolar CM [6].

CHF, especially in advanced stages of New York Heart Association (NYHA) class 3-4 contributes to the development of CIN primarily by decreasing renal perfusion. It is related with systolic dysfunction and low stroke volume [7]. In fact, some researches specifically pointed ejection fraction $(E F)<30-40 \%$ out as an independent predictor of CIN [8]. However, CHF per se and just a past history of heart failure increased the incidence of CIN, irrespective of EF $[9,10]$. Nevertheless, no studies have yet evaluated the association between diastolic dysfunction and CIN.

The golden standard estimating diastolic function of the heart is to measure left ventricular end-diastolic pressure (LVEDP) with catheterization. However, it is invasive and not a routine practice. On the other hand, E/ E' can be assessed non-invasively with echocardiography, and is known to be less influenced by heart rate, atrial activity, or ejection fraction [11]. In addition, E/E' levels were well correlated with LVEDP, when patients were categorized into 3 groups based on the E/E' values of 8 and 15 [12]. E/E' > 11 also predicted LVEDP > $15 \mathrm{mmHg}$ with a sensitivity of $75 \%$ and a specificity of $93 \%$ [13]. Among various doppler estimates of diastolic function such as E, E/A, decrease in E/A with the valsalva maneuver, deceleration time (DT), and pulmonary venous atrial reversal duration, E/E' showed the highest predictive power for LVEDP [12].

So, we aimed to investigate whether E/E' is an independent risk factor predicting the development of CIN. Effects of E/E' and CIN on patient mortality were also evaluated.

\section{Methods}

\section{Ethics statement}

This study was carried out in accordance with the Declaration of Helsinki and approved by the Institutional Review Board (IRB) of Yonsei University Health System Clinical Trial Center. Written consents were not required because this was a retrospective medical recordbased study and personally identifiable information was anonymized.

\section{Patients}

This retrospective study included 735 patients who underwent percutaneous transluminal coronary angioplasty (PTCA) at Severance Hospital in Seoul, Korea from January 2009 to December 2010. PTCA was conducted in 6837 patients for the following reasons: 1) regular follow-up for known coronary artery occlusive disease (CAOD), 2) suspicion for CAOD based on clinical symptoms or study results (treadmill test, technetium sestamibi scan, or cardiac CT or MRI), or 3) to evaluate whether idiopathic CHF was due to ischemia. Among these, 3302 subjects who had undergone echocardiography within 1 month of PTCA were included. Exposure to other CM within 7 days of PTCA ( $\mathrm{n}=$ 1576), an absence of data on serum creatinine during the 72 hours following the procedure $(\mathrm{n}=503)$, endstage renal disease $(n=408)$, underlying malignancy $(\mathrm{n}=43)$, acute infection $(\mathrm{n}=28)$, or age less than 18 years $(n=9)$ resulted in the exclusion of an additional 2567 patients.

All patients were hydrated with $0.9 \%$ saline at a rate of $1.0 \mathrm{~mL} / \mathrm{kg} / \mathrm{hour}$ for 12 hours pre- and post-exposure to the $\mathrm{CM}$, according to the guidelines of our clinic. In case of an emergency procedure, hydration was initiated immediately prior to the start of angiography and continued for 24 hours after PTCA. Infusion rate was reduced to $0.5 \mathrm{~mL} / \mathrm{kg} /$ hour when pulmonary edema was noted or ejection fraction was less than $30 \%$.

Non-ionic, dimeric, and iso-osmolar contrast agent (iodixanol: Visipaque, GE Healthcare, Amersham, United Kingdom) was used for PTCA. A hypotensive event was defined as systolic blood pressure $<80 \mathrm{mmHg}$ for at least 1 hour requiring inotropic therapy or IABP insertion within 24 hours of procedure, as described by Mehran et al. [14]. 


\section{Data collection}

Demographic and clinical data at the time of the PTCA were collected through medical chart review. Laboratory findings which were reported 24 hours prior to the PTCA and measured in overnight fasting status, were set as baseline values. CIN was defined as an increase of $\geq 0.5 \mathrm{mg} / \mathrm{dL}$ or $\geq 25 \%$ in serum creatinine level during the 72 hours following PTCA, that could not be better explained by alternative etiologies. We also used a second definition of CIN according to the Acute Kidney Injury Network (AKIN) criteria: a rise in serum creatinine $\geq 0.3 \mathrm{mg} / \mathrm{dL}$ within 48 hours of procedure $[3,15]$. Oliguria was not considered because all patients were hydrated and some of them used diuretics.

Hemoglobin, albumin, blood urea nitrogen, creatinine, serum cholesterol, triglycerides, and glucose levels were measured by an Advia 2120 Hematology Analyzer (Siemens Health-care Diagnostics, Deerfield, Illinois). High-sensitivity C-reactive protein (hs-CRP) levels were measured with a BN II analyzer (Dade Behring, Newark, DE, USA) using the latex-enhanced immunonephelometric method. The estimated glomerular filtration rate (eGFR) was calculated using the Modification of Diet in Renal Disease (MDRD) equation [16].

\section{Cardiac status and echocardiographic parameters}

Records of PTCA were reviewed for the urgency of the procedure, amount of CM used, interventional strategy, and the number of coronary arteries involved. We also investigated whether any patients had undergone coronary artery bypass graft surgery previously.

Echocardiography was performed with a SONOS 7500 (Philips Ultrasound, Bothell, WA, USA) according to the recommendations of the American Society of Echocardiography (ASE). Inter-ventricular septal thickness (IVSs, IVSd), posterior wall thickness (PWTs, PWTd), left ventricular end-diastolic dimension (LVDd), and left ventricular end-systolic dimension (LVDs) were measured in 2-dimensional $\mathrm{M}$ mode. Pulsed wave doppler was applied to check velocities in the 4-chamber apical view. Volume sampling was positioned at the tip of the mitral valve to measure early LV filling velocity (E) and left atrial contraction velocity (A). Tissue doppler was then conducted with the volume sampling repositioning at the septal annulus of the mitral valve to measure early (E') and late (A') diastolic mitral annular velocities.

LV systolic function was defined by EF. The EF was calculated by the modified Simpson's method, subtracting LVDs from LVDd. LV mass (LVM) was estimated using the Devereux modified ASE cube formula [17], and the LV mass index (LVMI) was calculated by dividing LVM by body surface area.

$$
\begin{aligned}
\text { LVM }=0.8 & \times[1.04 \times(\operatorname{IVSd}+\mathrm{LVDd} \\
& \left.+ \text { PWTd })^{3}-\mathrm{LVDd}^{3}\right]+0.6(\mathrm{gm})
\end{aligned}
$$

E/E' and E/A were calculated to represent diastolic function. DT, the time between peak $\mathrm{E}$ wave and the upper deceleration slope extrapolated to zero line, was also determined. In 23 patients exhibiting atrial fibrillation ( $\mathrm{n}=21,3.1 \%$ in non-CIN group and $\mathrm{n}=2,3.1 \%$ in CIN group), average measurements from 10 cardiac cycles were used [11].

Left atrial volume index (LAVI), a marker of volume status, was estimated with the biplane Simpson's method using the diameters of the LA, which were measured 3 times at the parasternal long axis view (anterior-posterior, superior-inferior) and the 4 chamber view (mediolateral).

Inter-reader reliability, intra-reader reliability, and reader drift analyses were performed on a random sample of $3 \%$ of the entire cohort. The intra-class correlation coefficients for the echocardiographic measures were 0.845 for EF, 0.765 for LVMI, and 0.753 for LAVI.

\section{Statistical analysis}

Statistical analysis was performed with the Statistical Package for the Social Sciences (SPSS) for Windows, version 18.0 (Chicago, IL, USA). Data are expressed as a mean \pm standard deviation for continuous variables, and as a number and percentage for categorical variables. Normality of distribution was examined by the ShapiroWilk test.

We compared the demographic, laboratory, and echocardiographic parameters of patients with CIN with those of patients without CIN using the Student's t-test, Mann-Whitney U test, or chi-square test. Either ANOVA or the Kruskal-Wallis test was performed to compare the 3 groups, which were classified according to the E/E' values of 8 and 15 . Univariate and multivariate binary logistic regression analyses were conducted to identify risk factors predicting the development of CIN. We also performed receiver operating characteristic (ROC) analysis to confirm the predictive accuracy of echocardiographic parameters for CIN. All-cause mortality was compared between patients using 4 groupings that were based on the presence or absence of diastolic dysfunction $\left(E / E^{\prime}>15\right)$ and CIN, by Kaplan-Meier and log-rank test. A p-value of less than 0.05 was considered statistically significant.

\section{Results}

Baseline characteristics between patients with CIN and those without CIN

Baseline characteristics of the study subjects are detailed in Table 1 . The mean age was $64.8 \pm 10.6$ years and $69.9 \%$ 
were male. The mean values of eGFR and E/E' were $74.5 \pm$ $20.5 \mathrm{~mL} / \mathrm{min} / 1.73 \mathrm{~m}^{2}$ and $13.3 \pm 5.3$, respectively. A total of 64 patients $(8.7 \%)$ developed CIN.

When patients were dichotomized into CIN and nonCIN groups, the CIN group was older and had lower body mass index (BMI) than the non-CIN group. The prevalence of hypertension, DM, emergency/ urgent procedure, three-vessel coronary artery disease (3-VD), previous coronary artery bypass graft surgery, and IABP use during the PTCA was significantly higher in the CIN

Table 1 Baseline characteristics of study subjects

\begin{tabular}{|c|c|c|c|c|}
\hline Variables & All $(n=735)$ & CIN $(n=64)$ & $\begin{array}{c}\text { No CIN } \\
(n=671)\end{array}$ & $p$ \\
\hline Age (years) & $64.8 \pm 10.6$ & $70.8 \pm 10.5$ & $64.2 \pm 10.5$ & $<0.001$ \\
\hline Sex (male) & $514(69.9 \%)$ & $39(60.9 \%)$ & $475(70.8 \%)$ & 0.101 \\
\hline BMI $\left(\mathrm{kg} / \mathrm{m}^{2}\right)$ & $24.5 \pm 3.3$ & $22.7 \pm 3.3$ & $24.7 \pm 3.3$ & $<0.001$ \\
\hline Systolic BP (mmHg) & $119.6 \pm 15.1$ & $119.5 \pm 21.6$ & $119.6 \pm 14.4$ & 0.979 \\
\hline Diastolic BP (mmHg) & $71.9 \pm 9.9$ & $69.0 \pm 14.2$ & $72.2 \pm 9.3$ & 0.119 \\
\hline Hypertension & $475(64.6 \%)$ & $52(81.3 \%)$ & $423(63.0 \%)$ & 0.004 \\
\hline Diabetes mellitus & $250(34.0 \%)$ & $39(60.9 \%)$ & $211(31.4 \%)$ & $<0.001$ \\
\hline Previous CABG & $23(3.1 \%)$ & $5(7.8 \%)$ & $18(2.7 \%)$ & 0.042 \\
\hline Emergency/ urgent procedure & $160(21.8 \%)$ & $23(35.9 \%)$ & $137(20.4 \%)$ & 0.004 \\
\hline Interventional strategy & & & & 0.521 \\
\hline Ballooning & $23(3.1 \%)$ & $1(1.6 \%)$ & $22(3.3 \%)$ & \\
\hline Ballooning + Stent insertion & $614(83.5 \%)$ & $52(81.3 \%)$ & $562(83.8 \%)$ & \\
\hline Ballooning + Stent insertion + Thrombus suction & $98(13.3 \%)$ & $11(17.2 \%)$ & $87(13.0 \%)$ & \\
\hline 3-vessel disease & $241(32.8 \%)$ & $39(60.9 \%)$ & $202(30.1 \%)$ & $<0.001$ \\
\hline Hypotensive event & $90(12.2 \%)$ & $25(39.1 \%)$ & $65(9.7 \%)$ & $<0.001$ \\
\hline IABP use & $32(4.4 \%)$ & $16(25.0 \%)$ & $16(2.4 \%)$ & $<0.001$ \\
\hline Total volume of $\mathrm{CM}(\mathrm{mL})$ & $237.0 \pm 74.5$ & $222.8 \pm 71.4$ & $238.1 \pm 74.7$ & 0.179 \\
\hline Volume of CM per weight (mL/kg) & $3.69 \pm 1.31$ & $4.09 \pm 1.86$ & $3.66 \pm 1.25$ & 0.179 \\
\hline N-acetylcysteine & $233(33.5 \%)$ & $15(42.9 \%)$ & $218(33.0 \%)$ & 0.230 \\
\hline BUN (mg/dL) & $18.0 \pm 7.3$ & $24.2 \pm 11.2$ & $17.4 \pm 6.6$ & $<0.001$ \\
\hline Creatinine $(\mathrm{mg} / \mathrm{dL})$ & $1.08 \pm 0.41$ & $1.50 \pm 0.75$ & $1.04 \pm 0.33$ & $<0.001$ \\
\hline $\operatorname{eGFR}\left(\mathrm{mL} / \mathrm{min} / 1.73 \mathrm{~m}^{2}\right)$ & $74.5 \pm 20.5$ & $54.5 \pm 25.8$ & $76.4 \pm 18.9$ & $<0.001$ \\
\hline Hemoglobin (g/dL) & $13.2 \pm 1.8$ & $11.5 \pm 2.1$ & $13.3 \pm 1.7$ & $<0.001$ \\
\hline Albumin (g/dL) & $4.31 \pm 0.48$ & $3.85 \pm 0.61$ & $4.35 \pm 0.44$ & $<0.001$ \\
\hline hs-CRP (mg/L) & $10.18 \pm 28.84$ & $34.11 \pm 47.95$ & $7.84 \pm 24.77$ & $<0.001$ \\
\hline Cholesterol (mg/dL) & $164.5 \pm 43.5$ & $167.5 \pm 56.5$ & $164.3 \pm 42.2$ & 0.722 \\
\hline Triglyceride (mg/dL) & $140.0 \pm 82.3$ & $126.0 \pm 87.7$ & $141.3 \pm 81.7$ & 0.035 \\
\hline Glucose (mg/dL) & $124.6 \pm 46.3$ & $156.2 \pm 71.3$ & $121.6 \pm 42.1$ & $<0.001$ \\
\hline Ejection fraction (\%) & $57.5 \pm 13.4$ & $48.8 \pm 12.8$ & $58.6 \pm 12.7$ & $<0.001$ \\
\hline$E / E^{\prime}$ & $13.3 \pm 5.3$ & $18.2 \pm 7.0$ & $12.9 \pm 4.9$ & $<0.001$ \\
\hline$E / A$ & $0.94 \pm 0.48$ & $0.95 \pm 0.37$ & $0.94 \pm 0.49$ & 0.630 \\
\hline Deceleration time (ms) & $204.6 \pm 50.1$ & $182.5 \pm 50.5$ & $206.7 \pm 49.7$ & $<0.001$ \\
\hline LV mass (g) & $213.6 \pm 95.7$ & $233.1 \pm 56.2$ & $211.9 \pm 98.4$ & 0.001 \\
\hline LV mass index $\left(\mathrm{g} / \mathrm{m}^{2}\right)$ & $126.3 \pm 88.6$ & $144.0 \pm 28.9$ & $124.7 \pm 91.9$ & $<0.001$ \\
\hline LVDd (mm) & $50.0 \pm 5.0$ & $51.2 \pm 5.6$ & $49.9 \pm 4.9$ & 0.030 \\
\hline LA volume index $\left(\mathrm{mL} / \mathrm{m}^{2}\right)$ & $28.3 \pm 9.7$ & $33.4 \pm 11.6$ & $27.8 \pm 9.4$ & $<0.001$ \\
\hline
\end{tabular}

Abbreviations: $B M I$ body mass index; $B P$ blood pressure; $B U N$ blood urea nitrogen; $C A B G$ coronary artery bypass graft; $C I N$ contrast-induced nephropathy; $C M$ contrast media; eGFR estimated glomerular filtration rate; $h s$-CRP high sensitivity C-reactive protein; IABP intra-aortic balloon pump; $L A$ left atrium; $L V$ left ventricle; LVDd left ventricular end-diastolic dimension. 
group compared to the non-CIN group. Within the laboratory findings, patients with CIN demonstrated eGFR, hemoglobin, and albumin levels that were significantly lower, while hs-CRP levels were significantly higher compared to the non-CIN group. Echocardiographic findings revealed that the EF and DT were decreased, while E/ E' $(18.2 \pm 7.0$ vs. $12.9 \pm 4.9, \mathrm{p}<0.001)$, LVMI, LVDd, and LAVI were significantly increased in patients with CIN.

Table 2 Differences in variables according to tertiles of $E / E^{\prime}$

\begin{tabular}{|c|c|c|c|c|}
\hline Variables & $\begin{array}{l}E / E^{\prime} \leq 8 \\
(n=47)\end{array}$ & $\begin{array}{c}8<E / E^{\prime} \leq 15 \\
(n=494)\end{array}$ & $\begin{array}{l}E / E^{\prime}>15 \\
(n=194)\end{array}$ & $p$ \\
\hline Age (years) & $57.0 \pm 9.5$ & $63.4 \pm 10.3$ & $70.1 \pm 9.6$ & 0.036 \\
\hline Sex (male) & $42(89.4 \%)$ & $375(75.9 \%)$ & $97(50.0 \%)$ & $<0.001$ \\
\hline BMI $\left(\mathrm{kg} / \mathrm{m}^{2}\right)$ & $23.7 \pm 2.3$ & $24.7 \pm 3.3$ & $24.4 \pm 3.6$ & 0.061 \\
\hline Systolic BP (mmHg) & $115.3 \pm 13.6$ & $119.9 \pm 14.0$ & $119.9 \pm 17.9$ & 0.018 \\
\hline Diastolic BP (mmHg) & $71.3 \pm 9.4$ & $72.6 \pm 9.4$ & $70.4 \pm 10.8$ & 0.150 \\
\hline Hypertension & $21(44.7 \%)$ & $305(61.7 \%)$ & $149(76.8 \%)$ & $<0.001$ \\
\hline Diabetes mellitus & $12(25.5 \%)$ & $154(31.2 \%)$ & $84(43.3 \%)$ & 0.005 \\
\hline Previous CABG & $0(0.0 \%)$ & $13(2.6 \%)$ & $10(5.2 \%)$ & 0.130 \\
\hline Emergency/ urgent procedure & $5(10.6 \%)$ & $103(20.9 \%)$ & $52(26.8 \%)$ & 0.038 \\
\hline Interventional strategy & & & & 0.680 \\
\hline Ballooning & $1(2.1 \%)$ & $14(2.8 \%)$ & $8(4.1 \%)$ & \\
\hline Ballooning + Stent insertion & $42(89.4 \%)$ & 415 (84.0\%) & $157(80.9 \%)$ & \\
\hline Ballooning + Stent insertion + Thrombus suction & $4(8.5 \%)$ & $65(13.2 \%)$ & $29(14.9 \%)$ & \\
\hline 3-vessel disease & $12(25.5 \%)$ & $134(27.1 \%)$ & $95(49.0 \%)$ & $<0.001$ \\
\hline Hypotensive event & $3(6.4 \%)$ & $45(9.1 \%)$ & $42(21.6 \%)$ & $<0.001$ \\
\hline IABP use & $0(0.0 \%)$ & $11(2.2 \%)$ & $21(10.8 \%)$ & $<0.001$ \\
\hline Total volume of $\mathrm{CM}(\mathrm{mL})$ & $236.6 \pm 89.4$ & $237.9 \pm 75.0$ & $234.6 \pm 69.1$ & 0.956 \\
\hline Volume of CM per weight $(\mathrm{mL} / \mathrm{kg})$ & $3.53 \pm 1.19$ & $3.62 \pm 1.22$ & $3.92 \pm 1.53$ & 0.176 \\
\hline N-acetylcysteine & $24(25.3 \%)$ & $144(33.2 \%)$ & $65(39.2 \%)$ & 0.071 \\
\hline $\mathrm{CIN}$ & $2(4.3 \%)$ & $20(4.0 \%)$ & $42(21.6 \%)$ & $<0.001$ \\
\hline BUN (mg/dL) & $15.6 \pm 3.5$ & $17.3 \pm 6.3$ & $20.2 \pm 9.6$ & 0.112 \\
\hline Creatinine (mg/dL) & $1.02 \pm 0.25$ & $1.04 \pm 0.32$ & $1.21 \pm 0.57$ & 0.848 \\
\hline eGFR $\left(\mathrm{mL} / \mathrm{min} / 1.73 \mathrm{~m}^{2}\right)$ & $82.9 \pm 17.9$ & $77.4 \pm 18.7$ & $64.9 \pm 22.4$ & 0.071 \\
\hline Hemoglobin (g/dL) & $13.9 \pm 1.3$ & $13.4 \pm 1.7$ & $12.3 \pm 1.9$ & 0.058 \\
\hline Albumin (g/dL) & $4.45 \pm 0.43$ & $4.39 \pm 0.41$ & $4.06 \pm 0.54$ & 0.308 \\
\hline hs-CRP (mg/L) & $6.85 \pm 29.80$ & $7.67 \pm 24.10$ & $17.20 \pm 36.50$ & $<0.001$ \\
\hline Cholesterol (mg/dL) & $161.7 \pm 42.7$ & $164.6 \pm 42.9$ & $165.4 \pm 45.7$ & 0.570 \\
\hline Triglyceride (mg/dL) & $143.9 \pm 95.3$ & $142.5 \pm 85.1$ & $132.4 \pm 70.4$ & 0.742 \\
\hline Glucose (mg/dL) & $116.6 \pm 41.3$ & $121.8 \pm 41.6$ & $133.7 \pm 56.6$ & 0.205 \\
\hline Ejection fraction (\%) & $61.0 \pm 10.0$ & $59.4 \pm 12.1$ & $52.7 \pm 14.4$ & 0.578 \\
\hline$E / E^{\prime}$ & $6.7 \pm 0.8$ & $11.2 \pm 2.1$ & $20.4 \pm 4.9$ & $<0.001$ \\
\hline$E / A$ & $0.98 \pm 0.38$ & $0.90 \pm 0.50$ & $1.01 \pm 0.44$ & 0.065 \\
\hline Deceleration time (ms) & $207.5 \pm 46.1$ & $207.9 \pm 49.5$ & $195.6 \pm 52.0$ & 0.699 \\
\hline LV mass (g) & $188.6 \pm 41.6$ & $212.1 \pm 108.2$ & $223.6 \pm 64.5$ & 0.014 \\
\hline LV mass index $\left(\mathrm{g} / \mathrm{m}^{2}\right)$ & $107.2 \pm 22.6$ & $121.5 \pm 57.0$ & $143.5 \pm 145.8$ & 0.003 \\
\hline LVDd (mm) & $48.4 \pm 3.5$ & $49.8 \pm 4.7$ & $50.8 \pm 5.8$ & 0.014 \\
\hline LA volume index $\left(\mathrm{mL} / \mathrm{m}^{2}\right)$ & $22.3 \pm 4.8$ & $26.6 \pm 7.9$ & $34.1 \pm 11.9$ & $<0.001$ \\
\hline
\end{tabular}

Abbreviations: $B M I$ body mass index; $B P$ blood pressure; $B U N$ blood urea nitrogen; $C A B G$ coronary artery bypass graft; $C I N$ contrast-induced nephropathy; $C M$ contrast media; eGFR estimated glomerular filtration rate; $h s$-CRP high sensitivity C-reactive protein; IABP intra-aortic balloon pump; $L A$ left atrium; $L V$ left ventricle; LVDd left ventricular end-diastolic dimension. 
When patients were classified into three groups based on the E/E' values of 8 and 15, CIN occurred in 42 (21.6\%) patients in the highest tertile compared with $20(4.0 \%)$ in the middle and $2(4.3 \%)$ in the lowest tertile $(\mathrm{p}<0.001)$. In addition, patients in the highest tertile were older and had more hypertension, DM, and 3-VD. They also experienced more hypotensive events and had IABPs placed more frequently during procedure than patients in the middle and lowest tertiles. Among the echocardiographic parameters, LVMI, LVDd, and LAVI were significantly increased in the highest tertile (Table 2).

\section{Risk factors for the development of CIN}

Logistic regression analysis showed that higher E/E' level [odds ratio (OR) 1.147, 95\% confidence interval (CI) 1.101$1.194, \mathrm{p}<0.001$ as a continuous variable/ OR 6.519, 95\% CI 3.774-11.260, $\mathrm{p}<0.001$ as a categorical variable] was a significant risk factor for CIN (Table 3). After adjustment for age, BMI, hypertension, DM, emergency/ urgent procedure, 3-VD, volume of CM per weight, use of IABP, eGFR $<60 \mathrm{~mL} / \mathrm{min} / 1.73 \mathrm{~m}^{2}$, hemoglobin, albumin, and hsCRP, E/E' still remained as an independent risk factor (OR $1.091,95 \%$ CI 1.026-1.159, $\mathrm{p}=0.005$ as a continuous variable/ OR 3.435, 95\% CI 1.522-7.755, p = 0.003 as a categorical variable). In successive models, further adjustments were made with other echocardiographic parameters, EF and LAVI. E/E' > 15 was found to be a final determinant of CIN (OR 2.579, 95\% CI 1.082-5.964, p = 0.035) (Table 4).

Defining CIN as an increase in serum creatinine $\geq 0.3$ $\mathrm{mg} / \mathrm{dL}$ from the baseline value, according to the AKIN criteria, did not change the independent role of E/E' for the prediction of CIN (OR 2.456, 95\% CI 1.046-6.217, p = 0.044) (see Additional file 1).

In subgroup analysis, CIN occurred most often in patients in the highest tertile of E/E' irrespective of diabetes status $(\mathrm{n}=15,13.6 \%$ in non-DM group, $\mathrm{p}<$ $0.001 / \mathrm{n}=27,32.1 \%$ in DM group, $\mathrm{p}<0.001)$. A similar finding was observed in patients with an eGFR $<60$ $\mathrm{mL} / \mathrm{min} / 1.73 \mathrm{~m}^{2}(\mathrm{n}=35,47.3 \%$ in the highest tertile of $\mathrm{E} / \mathrm{E}, \mathrm{p}<0.001)$, although there was no difference in the development of CIN among the three tertiles of E/E' in patients with an eGFR $\geq 60 \mathrm{~mL} / \mathrm{min} / 1.73 \mathrm{~m}^{2}(\mathrm{p}=0.199)$ (Figure 1).

\section{Receiver operating characteristic analysis of $E / E^{\prime}$ for the development of CIN}

To estimate the predictive accuracy of the echocardiographic parameters for the development of CIN, ROC analysis was performed. The area under the curve (AUC) for E/E, EF, and LAVI were 0.75 (95\% CI 0.68-0.82, p < 0.001 ), 0.74 (95\% CI 0.67-0.81, p < 0.001), and 0.66 (95\% CI $0.58-0.74, \mathrm{p}<0.001$ ), respectively (Figure 2).

Table 3 Univariate logistic regression analysis for contrast-induced nephropathy

\begin{tabular}{|c|c|c|c|}
\hline Variables & OR & $95 \% \mathrm{Cl}$ & $p$ \\
\hline Age $>75$ years (vs. $\leq 75$ years) & 2.837 & $1.038-1.097$ & $<0.001$ \\
\hline BMI $\left(\mathrm{kg} / \mathrm{m}^{2}\right)$ & 0.808 & $0.738-0.886$ & $<0.001$ \\
\hline Hypertension & 2.541 & $1.330-4.852$ & 0.005 \\
\hline Diabetes mellitus & 3.401 & $2.006-55.766$ & $<0.001$ \\
\hline Emergency/ urgent procedure & 2.187 & $1.269-3.767$ & 0.005 \\
\hline \multicolumn{4}{|l|}{ (vs. elective procedure) } \\
\hline 3-vessel disease (vs. < 3-vessel involvement) & 3.622 & $2.135-6.145$ & $<0.001$ \\
\hline IABP use (vs. non-IABP use) & 16.872 & $7.670-37.113$ & $<0.001$ \\
\hline Volume of CM per weight (mL/kg) & 1.250 & $1.024-1.526$ & 0.028 \\
\hline $\mathrm{eGFR}<60 \mathrm{~mL} / \mathrm{min} / 1.73 \mathrm{~m}^{2}$ & 11.343 & 6.434-19.997 & $<0.001$ \\
\hline \multicolumn{4}{|l|}{ (vs. eGFR $\geq 60 \mathrm{~mL} / \mathrm{min} / 1.73 \mathrm{~m}^{2}$ ) } \\
\hline Hemoglobin (g/dL) & 0.571 & $0.490-0.664$ & $<0.001$ \\
\hline Albumin (g/dL) & 0.179 & $0.111-0.289$ & $<0.001$ \\
\hline hs-CRP (mg/L) & 1.017 & $1.010-1.023$ & $<0.001$ \\
\hline$E / E^{\prime}$ & 1.147 & $1.101-1.194$ & $<0.001$ \\
\hline$E / E^{\prime}>15$ (vs. E/E' $\left.\leq 15\right)$ & 6.519 & $3.774-11.260$ & $<0.001$ \\
\hline$E F \leq 40 \%$ (vs. EF > 40\%) & 5.142 & $2.907-9.096$ & $<0.001$ \\
\hline LAVI $>35 \mathrm{~mL} / \mathrm{m}^{2}$ (vs. LAVI $\leq 35 \mathrm{~mL} / \mathrm{m}^{2}$ ) & 3.865 & $2.230-6.700$ & $<0.001$ \\
\hline
\end{tabular}

Data are presented as odds ratios (OR) and 95\% confidence intervals $(\mathrm{CI})$.

Abbreviations: $B M I$ body mass index; IABP intra-aortic balloon pump; $C M$ contrast media; eGFR estimated glomerular filtration rate; $h s$ - $C R P$, high sensitivity $C$-reactive protein; $E F$ ejection fraction; $L A V I$ left atrial volume index. 
Table 4 Odds ratios and $95 \%$ confidence intervals for contrast-induced nephropathy according to the $E / E^{\prime}$ levels (Multivariate logistic regression analysis)

\begin{tabular}{lccc}
\hline & \multicolumn{3}{c}{$\mathbf{E} / \mathbf{E}^{\prime}$} \\
\cline { 2 - 4 } & $\mathbf{O R}$ & $\mathbf{9 5 \% ~ C l}$ & $\boldsymbol{p}$ \\
\hline${ }^{\mathrm{a}}$ Model 1 & 1.091 & $1.026-1.159$ & 0.005 \\
${ }^{\mathrm{b}}$ Model 2 & 3.435 & $1.522-7.755$ & 0.003 \\
${ }^{\mathrm{c}}$ Model 3 & 3.344 & $1.456-7.682$ & 0.004 \\
${ }^{\mathrm{d}}$ Model 4 & 2.579 & $1.082-5.964$ & 0.035 \\
\hline
\end{tabular}

a Model 1 (odds ratio per 1 increase in $E / E^{\prime}$ ) : adjusted for age ( $>75$ years vs. $\leq$ 75 years), BMI, hypertension, diabetes, emergent/ urgent procedure, 3-vessel disease, IABP use, volume of CM per weight, eGFR $\left(<60 \mathrm{~mL} / \mathrm{min} / 1.73 \mathrm{~m}^{2}\right.$ vs. eGFR $\geq 60 \mathrm{~mL} / \mathrm{min} / 1.73 \mathrm{~m}^{2}$ ), hemoglobin, albumin, and hs-CRP.

${ }^{b}$ Model 2 (odds ratio for $E / E^{\prime}>15$ vs. E/E' $\leq 15$ ) : adjusted for age ( $>75$ years vs. $\leq 75$ years), BMl, hypertension, diabetes, emergent/ urgent procedure, 3 vessel disease, IABP use, volume of CM per weight, eGFR $\left(<60 \mathrm{~mL} / \mathrm{min} / 1.73 \mathrm{~m}^{2}\right.$ vs. eGFR $\geq 60 \mathrm{~mL} / \mathrm{min} / 1.73 \mathrm{~m}^{2}$ ), hemoglobin, albumin, and hs-CRP.

' Model 3 (odds ratio for $E / E^{\prime}>15$ vs. $E / E^{\prime} \leq 15$ ) : adjusted for Model 2 plus ejection fraction ( $\leq 40 \%$ vs. $>40 \%$ ).

${ }^{d}$ Model 4 (odds ratio for $E / E^{\prime}>15$ vs. E/E' $\leq 15$ ) : adjusted for Model 3 plus left atrial volume index (> 35 vs. $\leq 35$ ).

Abbreviations: $B M I$ body mass index; $I A B P$ intra-aortic balloon pump; $C M$ contrast media; eGFR estimated glomerular filtration rate; $h s-C R P$ high sensitivity C-reactive protein.

When AKIN criteria were applied, estimated AUC of E/E' for CIN was 0.79 (95\% CI 0.73-0.85, p < 0.001) (see Additional file 2).

\section{Survival analysis in CIN and diastolic dysfunction}

During a mean follow-up of 17.9 months, 30 patients died. The most common causes of death were cardiovascular disease $(n=16,53.5 \%)$, followed by infection $(n=12,40 \%)$.

Kaplan-Meier analysis showed higher mortality rates in patients who developed CIN ( $\mathrm{n}=18,28.1 \%$ vs. $\mathrm{n}=11$, $1.6 \%)$. In addition, there was a tendency toward higher mortality in those with diastolic dysfunction and CIN ( $\mathrm{n}=$ 14, 33.3\%) (Figure 3). The two-year survival rates were $98.1 \%$ in patients without CIN or diastolic dysfunction,
95.8\% in patients with diastolic dysfunction but no CIN, $72.6 \%$ in subjects with CIN and normal diastolic function, and $58.1 \%$ in patients with both CIN and diastolic dysfunction $(\mathrm{p}<0.001)$.

\section{Comparison of $E / E^{\prime}$ with previous risk scoring systems for CIN}

We reclassified our patients according to the risk stratification systems suggested by Mehran et al. [14] and Bartholomew et al. [4]. The incidence of CIN was well proportional to both risk scores. Predictive performances for CIN, estimated by AUC on ROC analysis, were about $80 \%$ with previous methods and $75 \%$ with E/E' (see Additional file 3).

\section{Discussion}

This study showed that patients with CIN exhibited a lower EF and higher E/E' and LAVI on echocardiography. In addition, the highest tertile of E/E' was associated with a significantly increased risk of CIN, beyond the well-known risk factors such as DM and renal failure. This implicates that diastolic dysfunction may be a useful parameter for predicting the development of CIN.

There are both patient-related and patient-unrelated factors in the risk stratification systems for CIN. Patientrelated factors include chronic kidney disease (CKD), DM, emergency/ urgent procedure, IABP use, CHF, age > 75 years, hypertension, anemia, a hypotensive episode, and LVEF $<40 \%$. Patient-unrelated factors include using ionic and hyper-osmolar contrast agent, and high volume of CM $[9,14]$. These factors play a role in the development of CIN primarily by reducing effective renal blood flow, consequently causing hypoxic change and the synthesis of reactive oxygen species. Development of CIN has also been attributed to increased sympathetic tone, reninangiotensin-aldosterone system (RAAS) activation, the
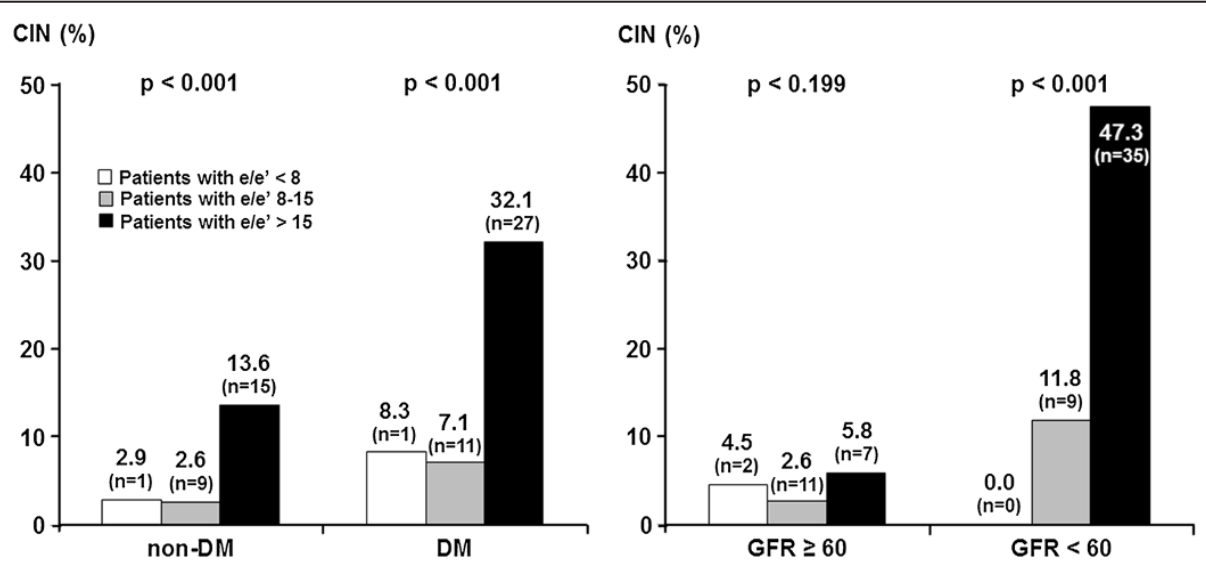

Figure 1 Relationship between diastolic dysfunction and the incidence of CIN according to the presence of diabetes or renal dysfunction. CIN developed most frequently in patients in the highest tertile of E/E' in all subgroups, except for patients with an eGFR $\geq$ $60 \mathrm{~mL} / \mathrm{min} / 1.73 \mathrm{~m}^{2}$ 


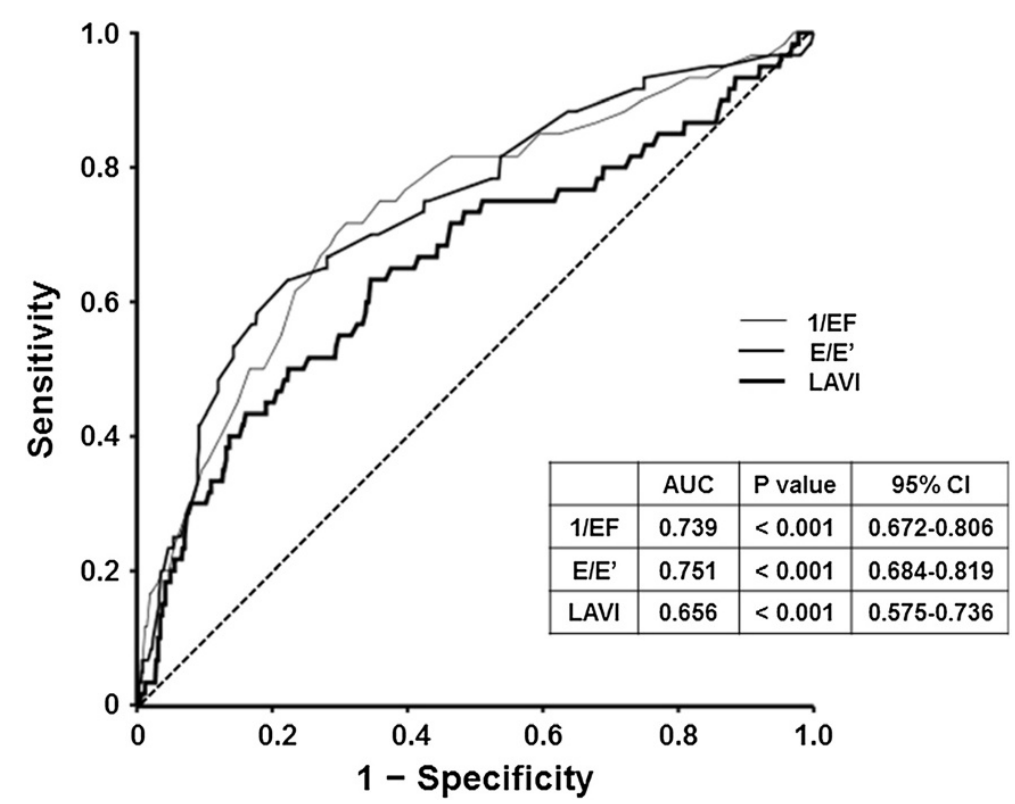

Figure 2 Receiver operating characteristic curves for the development of CIN according to the echocardiographic variables (EF, E/E', and LAVI). The AUCS of EF, E/E, and LAVI were $0.74,0.75$, and 0.66 , respectively $(p<0.001)$.

overproduction of many humoral factors such as vasopressin, catecholamines, endothelin, and pro-inflammatory cytokines, and decreased nitric oxide (NO) levels. These hemodynamic and neurohumoral alterations can cause vascular endothelial cell damage and hypertrophic change in the vascular smooth muscle cells, thus further aggravating blood flow disturbances [5,18].
Although CHF is a well-proven risk factor for CIN, only low ejection fraction has previously been studied, among various components of heart failure such as diastolic dysfunction, ventricular hypertrophy, and volume overload $[7,8,19]$. This has likely been because a decreased effective circulatory volume is considered the primary event in the development of CIN, in patients

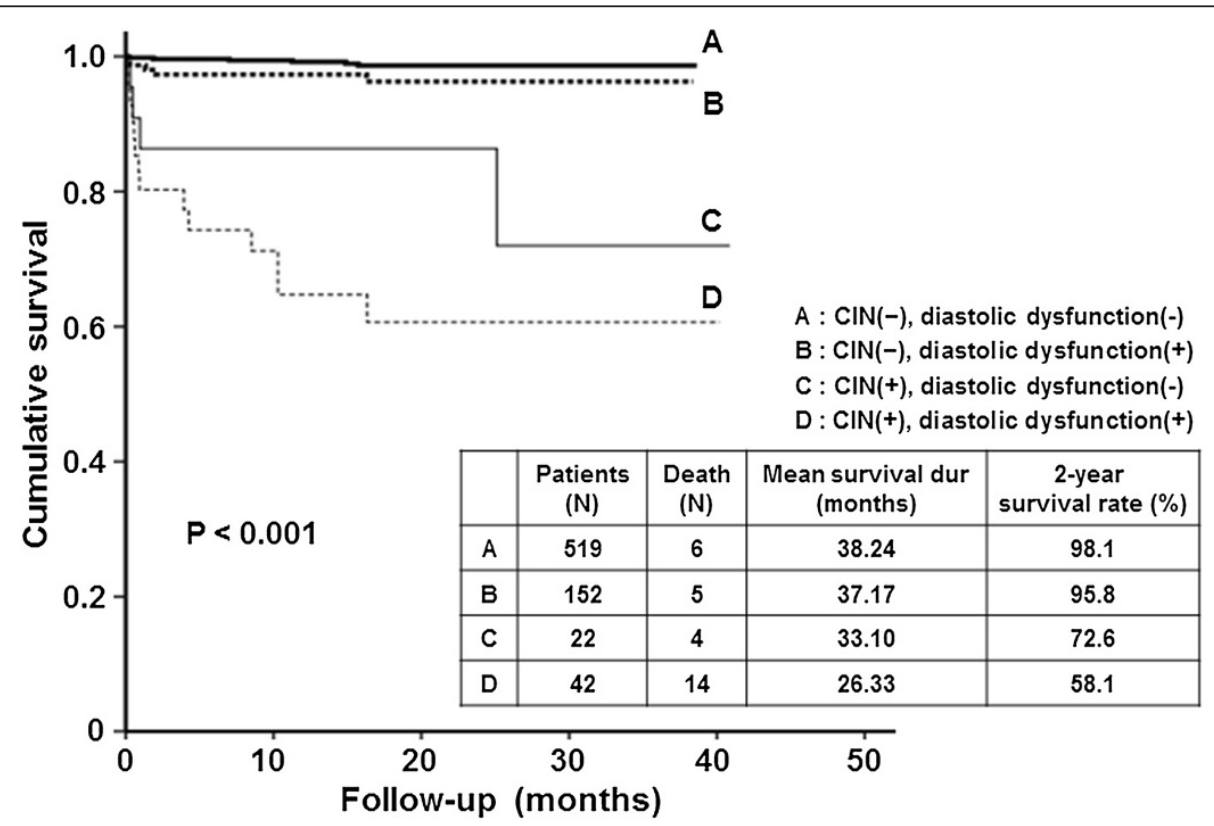

Figure 3 Kaplan-Meier analysis for cumulative hazard of patient mortality. There was a tendency toward higher mortality in patients with diastolic dysfunction (E/E' > 15) and/or CIN ( $<<0.001)$. 
with CHF [20,21]. However, the statistical significance of EF disappeared in the multivariate logistic regression analysis, whereas E/E' remained a significant risk factor for CIN (see Additional file 4). ROC analysis also showed a considerable predictive accuracy for E/E, which was comparable to that of EF (Figure 2). Moreover, most studies demonstrated that EF only affects CIN rates in patients with severe CHF of NYHA class 3-4 or an EF $<30-40 \%$. Given that CIN occurs in higher rates in CHF patients with lower severity, this suggests that the hemodynamic compromise status in milder stages of heart failure cannot be properly represented by EF [8]. Actually, CIN occurred in $18.2 \%$ of patients with $\mathrm{EF}<40 \%$, and $7.8 \%$ of patients with $\mathrm{EF} \geq 40 \%$ in our study. However, when patients with severe CHF were excluded, E/E' still was an independent risk factor for CIN (OR 2.593, 95\% CI 1.014-6.633, p = 0.041 , data not shown). This finding suggests that diastolic dysfunction may be a more reliable parameter to represent the hemodynamic and neurohumoral alterations observed in CHF.

Studies conducted in patients with $\mathrm{CHF}$ and preserved LVEF showed a proportional increase in renal failure according to the diastolic dysfunction [22]. The stiffer the LV, the faster the eGFR declined [23]. The main reasons for this include reduced LV functional reserve, resting/ exercise-exacerbated systolic dysfunction, and chronotropic incompetence, which are responsible for the decrease in blood flow. These result in insufficient tissue perfusion and an ischaemic injury to the kidney [24-26]. Initially, LV hypertrophy occurs to compensate for the stiffness of the LV, by increasing stroke volume. But pathologic proliferation, fibrotic deposition, and calcification of the ventricle become evident, and LV compliance eventually decreases [27]. The same vicious cycle in hemodynamics is thought to be the chief mechanism for a higher E/E' causing CIN. The adverse effect of diastolic dysfunction on patient mortality is partly attributed to these unfavorable geometric changes in the LV (Figure 3).

On the other hand, the neurohumoral changes occurring during the development of CIN further aggravate diastolic dysfunction. Angiotension II and aldosterone promote the growth/ proliferation of both cardiomyocytes and non-myocyte cells like fibroblasts [28]. Pro-inflammatory cytokines such as TNF- $\alpha$ and IL- 6 stimulate collagen production by fibroblasts, and bring about a myocardiodepressive effect [29]. We also demonstrated elevated hs-CRP levels in patients in the highest tertile of E/E' (Table 2). In addition, increased sympathetic tone activates the $\beta$-catenin pathway in the cardiomyocytes through the recruitment of Akt (protein kinase B). This up-regulates the production of osteoblastogenic proteins, further aggravating diastolic function [30]. Through these mutual effects on hemodynamic and neurohumoral status, higher $E / E^{\prime}$ is thought to cause acute kidney injury when patients with CHF are exposed to the CM.

Furthermore, it should be noted that older age and increased prevalences of hypertension, DM, and 3-VD were present in subjects with diastolic dysfunction (Table 2). These comorbidities are well-proven risk factors, and may potentiate the relationship between diastolic dysfunction and CIN. In DM, advanced glycation endproduct (AGE) mediates the crosslinking of collagen fibres in the myocardium [31]. It also causes an inflammation in the renal vascular system after binding to the receptor of AGE (RAGE), through activation of NADPH oxidase, MAP kinase, and the NF- $\mathrm{KB}$ pathway [32,33]. Because insulin and c-peptide are known to evoke the overexpression of inducible endothelial NO synthase, impaired pancreatic secretion is also expected to increase E/E' and exacerbate renal hemodynamics [34,35]. Actually, several studies demonstrated an improved ejection fraction/ diastolic function after correcting for these metabolic abnormalities in diabetic CKD patients with a kidney-pancreas co-transplantation [36,37]. Underlying hypertension and CKD also strengthen the linkage between diastolic dysfunction and CIN through the effects of uremic toxin [38,39], pressure overload, RAAS activation, and markedly elevated expression of humoral mediators such as catecholamines, endothelin, and parathyroid hormone [40].

There are several shortcomings to our study. Because this was a retrospective study, limited data were available, which may have affected the conclusion by type II error. Second, as estimating LVEDP during the PTCA was not a routine practice in our institute, LVEDP and their relationships with E/E' and CIN could not be presented. However, in other small cohort of 55 patients who measured LVEDP during the PTCA in our hospital, E/E' levels were well correlated with LVEDP $(r=0.78$, $\mathrm{p}<0.001)$. The ROC analysis demonstrated that the predictive accuracy of E/E' for LVEDP > $15 \mathrm{mmHg}$ was 0.88 ( $\mathrm{p}<0.001,95 \%$ CI 0.794-0.981). E/E' > 15 had $81 \%$ sensitivity and $86 \%$ specificity for LVEDP > $15 \mathrm{mmHg}$ (see Additional file 5). These results correspond closely with previous studies by Sohn et al. and Ommen et al. [11-13]. Moreover, E/E' can be measured non-invasively prior to PTCA, and warns the physicians to estimate LVEDP during procedure and to monitor patients' renal function carefully after procedure, in advance, when levels are elevated. Third, as only patients who were suspected to have CAOD and had undergone an echocardiography were included, selection bias may have existed. Higher incidence rate of CIN in our study group compared to that in previous studies conducted in the general population supports this [1]. However, considering that interventional procedures using intravascular $\mathrm{CM}$ are most closely related with the development of 
$\mathrm{CIN}$, and most patients requiring such procedures have underlying cardiac disease and DM, the results of present study can be applied to the high-risk patients planning for the angioplasty. The uncontrolled use of medications with vasomotor action or that modulate the neurohumoral axis is another limitation. However, former clinical trials using RAAS blockade, statins, calcium channel blockers, beta-blockers, and diuretics failed to derive a common consensus as to their effects on CIN [41-43]. Lastly, as it was a single-center study, further large-scale, randomized controlled multicenter trials are needed to confirm and assess the clinical applicability of our findings.

\section{Conclusions}

In conclusion, we demonstrated that, among echocardiographic parameters, E/E' can be a useful predictor for the development of CIN. To our knowledge, this is the first study suggesting that assessment of diastolic dysfunction should play a role in the risk stratification for CIN.

\section{Additional file}

\section{Additional file 1: Univariate logistic regression analysis for contrast-induced nephropathy, which was defined according to the AKIN criteria.}

Additional file 2: Receiver operating characteristic curves for CIN, which was defined according to the AKIN criteria. The AUCS of EF, $E / E$, and LAVI were $0.70,0.79$, and 0.69 , respectively $(p<0.001)$.

Additional file 3: Predictive performances for CIN using various risk scoring systems. The incidence of CIN was well proportional to both (a) Mehran's (renal failure was scored according to the eGFR: white bar, or the $\mathrm{Cr}$ levels: black bar) and (b) Bartholomew's risk scores. (c) E/E' showed a considerable predictive power for the development of $\mathrm{CIN}$, which was comparable to other risk stratification methods. The AUCS of E/E', Mehran's score, and Bartholomew's score were $0.75,0.80$, and 0.82 , respectively $(p<0.001)$.

Additional file 4: Multivariate logistic regression analysis for contrast-induced nephropathy (detailed descriptions of Table 4).

Additional file 5: Correlation between E/E' and LVEDP. (a) E/E' showed a significant positive relationship with LVEDP on correlation analysis. (b) Across increasing E/E' tertiles, LVEDP levels were incrementally higher. (c) ROC analysis revealed that the predictive accuracy of E/E' for LVEDP $>15 \mathrm{mmHg}$ was $0.88(p<0.001,95 \% \mathrm{Cl}$ 0.794-0.981)

\footnotetext{
Abbreviations

AGE: Advanced glycation endproduct; AKIN: Acute Kidney Injury Network; ANP: Atrial natriuretic peptide; ASE: American Society of Echocardiography; AUC: Area under the curve; BMI: Body mass index; CAOD: Coronary artery occlusive disease; CHF: Congestive heart failure; Cl: Confidence interval; CIN: Contrast-induced nephropathy; CKD: Chronic kidney disease; CM: Contrast media; DT: Deceleration time; EF: Ejection fraction; eGFR: Estimated glomerular filtration rate; Hs-CRP: High-sensitivity C-reactive protein; IABP: Intra-aortic balloon pump; IVS: Interventricular septal thickness; LAVI: Left atrial volume index; LVDd: Left ventricular end-diastolic dimension; LVDs: Left ventricular end-systolic dimension; LVEDP: Left ventricular enddiastolic pressure; LVM: Left ventricular mass; LVMI: Left ventricular mass index; MDRD: Modification of Diet in Renal Disease; NO: Nitric oxide; NYHA: New York Heart Association; OR: Odds ratio; PTCA: Percutaneous transluminal coronary angioplasty; PWT: Posterior wall thickness;
}

RAAS: Renin-angiotensin-aldosterone system; ROC: Receiver operating characteristic; 3-VD: Three-vessel coronary artery disease.

\section{Competing interests}

The authors declare that they have no competing interests.

\section{Authors' contributions}

HMK designed the study, acquired the data, performed the statistical analysis, interpreted the analytical results, and wrote the manuscript; $\mathrm{SHH}$, THY and SWK edited and made critical revision to the manuscript; HJO assisted in the study design and helped draft the manuscript; BSK participated in the data analysis and interpretation; FMD, KIK, CHK, and MJL assisted in the study design and contributed to the data acquisition; KHC organized the data collection and edited the manuscript. All authors approved the final version of the manuscript.

Received: 3 October 2012 Accepted: 8 July 2013

Published: 13 July 2013

\section{References}

1. Goldfarb S, McCullough PA, McDermott J, Gay SB: Contrast-induced acute kidney injury: specialty-specific protocols for interventional radiology, diagnostic computed tomography radiology, and interventional cardiology. Mayo Clin Proc Mayo Clin 2009, 84:170-179.

2. Finn WF: The clinical and renal consequences of contrast-induced nephropathy. Nephrol, Dial, Transplantat 2006, 21:i2-10.

3. McCullough PA: Contrast-induced acute kidney injury. J Am Coll Cardiol 2008, 51:1419-1428.

4. Bartholomew BA, Harjai KJ, Dukkipati S, Boura JA, Yerkey MW, Glazier S, Grines $C L$, O'Neill WW: Impact of nephropathy after percutaneous coronary intervention and a method for risk stratification. Am J Cardiol 2004, 93:1515-1519.

5. Goldenberg I, Matetzky S: CMAJ: Can Med Assoc J = Journal de l'Association Medicale Canadienne 2005, 172:1461-1471.

6. Mehran R, Nikolsky E: Contrast-induced nephropathy: definition, epidemiology, and patients at risk. Kidney Int Supp/ 2006, 100:11-15.

7. Rosenstock JL, Gilles E, Geller AB, Panagopoulos G, Mathew S, Malieckal D, DeVita MV, Michelis MF: Impact of heart failure on the incidence of contrast-induced nephropathy in patients with chronic kidney disease. Int Urol Nephrol 2010, 42:1049-1054.

8. Nikolsky E, Mehran R, Turcot D, Aymong ED, Mintz GS, Lasic Z, Lansky AJ, Tsounias E, Moses JW, Stone GW, et al: Impact of chronic kidney disease on prognosis of patients with diabetes mellitus treated with percutaneous coronary intervention. Am J Cardiol 2004, 94:300-305.

9. Pannu N, Wiebe N, Tonelli M: Prophylaxis strategies for contrast-induced nephropathy. JAMA 2006, 295:2765-2779.

10. Barrett BJ, Parfrey PS: Clinical practice. Preventing nephropathy induced by contrast medium. New Engl J Med 2006, 354:379-386.

11. Nagueh SF, Appleton CP, Gillebert TC, Marino PN, Oh JK, Smiseth OA, Waggoner AD, Flachskampf FA, Pellikka PA, Evangelisa A:

Recommendations for the evaluation of left ventricular diastolic function by echocardiography. Eur J Echocardiogr 2009, 10:165-193.

12. Ommen SR, Nishimura RA, Appleton CP, Miller FA, Oh JK, Redfield MM, Tajik AJ: Clinical utility of Doppler echocardiography and tissue Doppler imaging in the estimation of left ventricular filling pressures: A comparative simultaneous Doppler-catheterization study. Circulation 2000, 102:1788-1794.

13. Sohn DW, Song JM, Zo JH, Chai IH, Kim HS, Chun HG, Kim HC: Mitral annulus velocity in the evaluation of left ventricular diastolic function in atrial fibrillation. J Am Soc Echocardiogr 1999, 12:927-931.

14. Mehran R, Aymong ED, Nikolsky E, Lasic Z, lakovou I, Fahy M, Mintz GS, Lansky AJ, Moses JW, Stone GW, et al: A simple risk score for prediction of contrast-induced nephropathy after percutaneous coronary intervention: development and initial validation. J Am Coll Cardiol 2004, 44:1393-1399.

15. Mehta RL, Kellum JA, Shah SV, Molitoris BA, Ronco C, Warnock DG, Levin A: Acute Kidney Injury Network: report of an initiative to improve outcomes in acute kidney injury. Crit Care 2007, 11:R31.

16. Levey AS, Bosch JP, Lewis JB, Greene T, Rogers N, Roth D: A more accurate method to estimate glomerular filtration rate from serum creatinine: a new prediction equation. Modification of Diet in Renal Disease Study Group. Ann Intern Med 1999, 130:461-470. 
17. Devereux RB, Alonso DR, Lutas EM, Gottlieb GJ, Campo E, Sachs I, Reichek N: Echocardiographic assessment of left ventricular hypertrophy: comparison to necropsy findings. Am J Cardiol 1986, 57:450-458.

18. Tumlin J, Stacul F, Adam A, Becker CR, Davidson C, Lameire N, McCullough PA: Pathophysiology of contrast-induced nephropathy. Am J Cardiol 2006, 98:14K-20K.

19. Dangas G, lakovou I, Nikolsky E, Aymong ED, Mintz GS, Kipshidze NN, Lansky AJ, Moussa I, Stone GW, Moses JW, et al: Contrast-induced nephropathy after percutaneous coronary interventions in relation to chronic kidney disease and hemodynamic variables. Am J Cardiol 2005, 95:13-19.

20. Heyman SN, Rosenberger C, Rosen S: Regional alterations in renal haemodynamics and oxygenation: a role in contrast medium-induced nephropathy. Nephrol, Dial, Transplant 2005, 20(Suppl 1):i6-11.

21. Gami AS, Garovic VD: Contrast nephropathy after coronary angiography. Mayo Clin Proc Mayo Clin 2004, 79:211-219.

22. Victor BM, Barron JT: Diastolic heart failure versus diastolic dysfunction: difference in renal function. Clin Cardiol 2010, 33:770-774

23. de Sa DD C, Hodge DO, Slusser JP, Redfield MM, Simari RD, Burnett JC, Chen $\mathrm{HH}$ : Progression of preclinical diastolic dysfunction to the development of symptoms. Heart 2010, 96:528-532

24. Borlaug BA, Olson TP, Lam CS, Flood KS, Lerman A, Johnson BD, Redfield MM: Global cardiovascular reserve dysfunction in heart failure with preserved ejection fraction. J Am Coll Cardiol 2010, 56:845-854.

25. Borlaug BA, Paulus WJ: Heart failure with preserved ejection fraction: pathophysiology, diagnosis, and treatment. Eur Heart J 2011, 32:670-679.

26. Phan TT, Abozguia K, Nallur Shivu G, Mahadevan G, Ahmed I, Williams L, Dwivedi G, Patel K, Steendijk P, Ashrafian $\mathrm{H}$, et al: Heart failure with preserved ejection fraction is characterized by dynamic impairment of active relaxation and contraction of the left ventricle on exercise and associated with myocardial energy deficiency. J Am Coll Cardiol 2009, 54:402-409.

27. Afshinnia F, Spitalewitz S, Chou SY, Gunsburg DZ, Chadow HL: Left ventricular geometry and renal function in hypertensive patients with diastolic heart failure. Am J Fidney Dis 2007, 49:227-236.

28. Gajarsa JJ, Kloner RA: Left ventricular remodeling in the post-infarction heart: a review of cellular, molecular mechanisms, and therapeutic modalities. Heart Fail Rev 2011, 16:13-21.

29. Melendez GC, McLarty JL, Levick SP, Du Y, Janicki JS, Brower GL: Interleukin 6 mediates myocardial fibrosis, concentric hypertrophy, and diastolic dysfunction in rats. Hypertension 2010, 56:225-231.

30. Fang D, Hawke D, Zheng Y, Xia Y, Meisenhelder J, Nika H, Mills GB, Kobayashi R, Hunter T, Lu Z: Phosphorylation of beta-catenin by AKT promotes beta-catenin transcriptional activity. J Biol Chem 2007 282:11221-11229.

31. Hartog JW, Voors AA, Bakker SJ, Smit AJ, Van Veldhuisen DJ: Advanced glycation end-products (AGEs) and heart failure: pathophysiology and clinical implications. Eur J Heart Fail 2007, 9:1146-1155.

32. Basta G, Schmidt AM, De Caterina R: Advanced glycation end products and vascular inflammation: implications for accelerated atherosclerosis in diabetes. Cardiovasc Res 2004, 63:582-592.

33. Meerwaldt R, Links T, Zeebregts C, Tio R, Hillebrands JL, Smit A: The clinical relevance of assessing advanced glycation endproducts accumulation in diabetes. Cardiovasc Diabetol 2008, 7:29.

34. Kamikawa A, Ishii T, Shimada K, Makondo K, Inanami O, Sakane N, Yoshida T, Saito M, Kimura K: Proinsulin C-peptide abrogates type-1 diabetes-induced increase of renal endothelial nitric oxide synthase in rats. Diabetes Metab Res Rev 2008, 24:331-338.

35. Nordquist L, Brown R, Fasching A, Persson P, Palm F: Proinsulin C-peptide reduces diabetes-induced glomerular hyperfiltration via efferent arteriole dilation and inhibition of tubular sodium reabsorption. Am J Physiol Renal Physiol 2009, 297:F1265-1272.

36. Fiorina P, La Rocca E, Astorri E, Lucignani G, Rossetti C, Fazio F, Giudici D, Di Carlo V, Cristallo M, Pozza G, Secchi A: Reversal of left ventricular diastolic dysfunction after kidney-pancreas transplantation in type 1 diabetic uremic patients. Diabetes Care 2000, 23:1804-1810.

37. Perseghin G, Fiorina P, De Cobelli F, Scifo P, Esposito A, Canu T, Danna M, Gremizzi C, Secchi A, Luzi L, Del Maschio A: Cross-sectional assessment of the effect of kidney and kidney-pancreas transplantation on resting left ventricular energy metabolism in type 1 diabetic-uremic patients: a phosphorous-31 magnetic resonance spectroscopy study. J Am Coll Cardiol 2005, 46:1085-1092
38. Stewart GA, Gansevoort RT, Mark PB, Rooney E, McDonagh TA, Dargie HJ Stuart R, Rodger C, Jardine AG: Electrocardiographic abnormalities and uremic cardiomyopathy. Kidney Int 2005, 67:217-226.

39. Lekawanvijit S, Adrahtas A, Kelly DJ, Kompa AR, Wang BH, Krum H: Does indoxyl sulfate, a uraemic toxin, have direct effects on cardiac fibroblasts and myocytes? Eur Heart J 2010, 31:1771-1779.

40. Dickhout JG, Carlisle RE, Austin RC: Interrelationship between cardiac hypertrophy, heart failure, and chronic kidney disease: endoplasmic reticulum stress as a mediator of pathogenesis. Circ Res 2011, 108:629-642.

41. Zhang L, Lu Y, Wu B, Zhang S, Jiang H, Ge J, Chen H: Efficacy of statin pretreatment for the prevention of contrast-induced nephropathy: a meta-analysis of randomised controlled trials. Int J Clin Pract 2011 65:624-630

42. Gleeson TG, Bulugahapitiya S: Contrast-induced nephropathy. AJR Am J Roentgenol 2004, 183:1673-1689.

43. Kiski D, Stepper W, Brand E, Breithardt G, Reinecke H: Impact of renin-angiotensin-aldosterone blockade by angiotensin-converting enzyme inhibitors or AT-1 blockers on frequency of contrast medium-induced nephropathy: a post-hoc analysis from the Dialysis-versus-Diuresis (DVD) trial. Nephrol, Dial, Transplant 2010, 25:759-764.

doi:10.1186/1471-2369-14-146

Cite this article as: Koo et al:: Diastolic dysfunction is associated with an increased risk of contrast-induced nephropathy: a retrospective cohort study. BMC Nephrology 2013 14:146.

\section{Submit your next manuscript to BioMed Central and take full advantage of:}

- Convenient online submission

- Thorough peer review

- No space constraints or color figure charges

- Immediate publication on acceptance

- Inclusion in PubMed, CAS, Scopus and Google Scholar

- Research which is freely available for redistribution
C) Biomed Central 